PREHOSPITAL CARE

\title{
Limited benefits of ambulance telemetry in delivering early thrombolysis: a randomised controlled trial
}

\author{
M Woollard, K Pitt, A J Hayward, N C Taylor
}

Emerg Med J 2005;22:209-215. doi: 10.1136/emj.2003.013482

See end of article for authors' affiliations

Correspondence to: Mr M Woollard, Faculty of Pre-hospital Care Research Unit, Department of Academic Emergency Medicine Academic Centre, The James Cook University Hospital, Marton Road, Middlesbrough TS4 3BW UK; malcolm.woollard@ ukgateway.net

Accepted 26 August 2004
Objectives: To evaluate the potential of a continuous telemetry system linking rural ambulances to a coronary care unit to reduce call to thrombolysis times.

Methods: This prospective randomised controlled trial recruited patients using the 999 ambulance service in a rural area of the UK with signs or symptoms of coronary heart disease. Subjects were assigned to receive either standard paramedic treatment or transmission of 12 lead ECG, blood pressure, pulse oximetry, and relevant medical history to a general hospital coronary care unit. Cardiology senior house officers then determined each patient's suitability for pre-hospital thrombolysis time, and transmitted this decision back to the ambulance. This was documented as the potential thrombolysis, although no thrombolytic agents were administered by paramedics. The between groups difference in time to potential thrombolysis (intervention group) and actual thrombolysis (controls) was compared. The proportion of intervention group subjects ultimately receiving thrombolysis in hospital was compared with that recommended for pre-hospital thrombolysis.

Results: The potential reduction in call to treatment time for telemetry patients recommended for prehospital thrombolysis was 55 minutes $(p=0.022)$. Following hospital admission, $21 / 213$ of the telemetry patients were thrombolysed (10\%, 95\% confidence interval (CI) $6 \%$ to 15\%). Of these patients, $3 / 21$ received a recommendation for thrombolysis in the ambulance $(14 \%, 95 \% \mathrm{Cl} 3.1 \%$ to $36.3 \%)$. The sensitivity and specificity of the telemetry system in detecting patients requiring thrombolysis was 13.6 and 99.5\% respectively. Errors were made in the pre-hospital treatment recommendations for two patients. Conclusions: Continuous telemetry systems may significantly reduce call to treatment times for patients recommended for pre-hospital thrombolysis in a rural setting. However, this benefit must be balanced against the very small proportion of eligible patients identified as suitable for pre-hospital thrombolysis. This limitation may be due to communications problems, the criteria used to identify eligible patients, or the seniority of physicians tasked with making treatment decisions.
$\mathrm{T}$ he efficacy of thrombolysis is related to the rapidity with which it is administered. A meta-analysis of 22 random-

ised trials comparing fibrinolytic therapy with placebo or controls reported that administration of a thrombolytic within 1 hour of symptom onset resulted in 65 lives saved per 1000 patients treated, but that the benefit tailed off appreciably thereafter. ${ }^{1}$ In the UK, a target call to thrombolysis time of 60 minutes has been established as government policy. ${ }^{23}$

Paramedics in the USA have a high success rate in acquiring diagnostic quality 12 lead ECGs from stable chest pain patients. ${ }^{4}$ Paramedic administered thrombolytic therapy following ECG transmission to physicians for a pre-hospital treatment recommendation has been shown to be feasible and safe..$^{5-7}$

Our trial evaluated the potential of a continuous telemetry system linking rural ambulances to a coronary care unit to reduce call to thrombolysis times in the UK.

\section{METHODS \\ Protocol}

This prospective randomised controlled trial was conducted within the catchment area of the coronary care unit (CCU) of one general hospital in a rural UK area between January 2001 and July 2002. We recruited patients with chest pain believed to be of cardiac origin or without a clear alternative cause; an abnormal 3 lead ECG and cardiovascular compromise (with or without chest pain); or those referred by a GP with a provisional diagnosis of a cardiac condition.

Telemetry group patients received standard pre-hospital treatment, including direct admission to the CCU, plus continuous transmission of 12 lead ECG, pulse oximetry, and blood pressure, while the ambulance was on route to the hospital. Relevant medical history was also transmitted after being obtained in accordance with 17 standardised questions addressing eligibility for pre-hospital thrombolysis, adapted from recommendations made by the Joint Royal Colleges Ambulance Liaison Committee (fig 1). A cardiology senior house officer used this data to make recommendations about suitability for pre-hospital thrombolysis. These were transmitted to ambulances as text messages, and the time of receipt noted as the time at which a thrombolytic could potentially have been given in the ambulance. The time of thrombolysis in the CCU was recorded, if appropriate.

Patients in the control group received standard treatment. If appropriate, the time of thrombolysis in the CCU was recorded. Fig 2 illustrates the study recruitment process.

The six ambulances in the study area were fitted with Mobimed telemetry units (Ortivus UK Ltd, Fareham, Hampshire, UK), providing continuous transmission to a computer receiving station in the CCU via a mobile phone. Two way, text based communication between paramedics and physicians was possible, but vocal contact was not.

Paramedics were provided with 18 hours of training in 12 lead ECG recording, the telemetry equipment, the study protocol, and how to succinctly elicit answers to thrombolysis eligibility questions. Physicians and CCU staff were trained to use the hospital receiving station and the study protocol. A 6 month pilot study was conducted to ensure staff could use the equipment effectively and to test the research protocol.

Abbreviations: $\mathrm{CCU}$, coronary care unit; $\mathrm{SHO}$, senior house officer 
- Confirm that the patient is conscious, coherent, and able to understand that clot dissolving drugs will be used.

- Confirm that the patient is aged 75 years or less.

- Confirm that the patient has had symptoms characteristic of a coronary heart attack (i.e. pain in a typical distribution of 30 minutes duration or more)

- Confirm that the continuous symptoms started less than 6 hours ago.

- Confirm that the pain built up over seconds and minutes rather than starting totally abruptly.

- Confirm that breathing does not influence the severity of the pain.

- Confirm that the heart rate is between 50-140.

- Confirm that the systolic blood pressure is more than $80 \mathrm{~mm} \mathrm{Hg}$ and less than $160 \mathrm{~mm} \mathrm{Hg}$.

- Confirm that the patient is not likely to be pregnant, nor has delivered within the last two weeks.

- Confirm that the patient has not had a peptic ulcer within the last 6 months.

- Confirm that the patient has not had a stroke of any sort within the last 12 months and no permanent disability from a previous stroke.

- Confirm that the patient has not been treated recently for any other serious brain condition? (This is intended to exclude patients with cerebral tumours).

- Confirm the patient has no diagnosed bleeding tendency, has had no recent blood loss (except for normal menstruation), and is not taking warfarin (anticoagulant) therapy.

- Confirm the patient has not had any surgical operation, tooth extractions, significant trauma, or head injury within the last 4 weeks.

- Confirm that streptokinase has not been given previously. (If the patient has had thrombolytic treatment and does not know which agent was used, you should assume that it was streptokinase).

- Confirm that the patient has not had chest compression for resuscitation for a period of longer than 5 minutes.

- Confirm that the patient is not being treated for liver failure, renal failure, or any other severe systemic illness.

Figure 1 Modified Joint Royal Colleges Ambulance Liaison Committee criteria for pre-hospital thrombolysis.

The primary outcome measure was a between groups comparison of call to potential or actual thrombolysis time. This was defined as the interval from the time of receipt of the 999 call to the time a thrombolytic was administered in the CCU (control group) or a recommendation was received for pre-hospital thrombolysis (telemetry group). The proportion of patients recommended for pre-hospital thrombolysis was also recorded. Patients in the telemetry group were followed up to determine if the pre-hospital thrombolysis recommendation accorded with that subsequently made in the CCU.

Ethics approval for the study was obtained from the DyfedPowys local research ethics committee. The investigators and the committee agreed that attempting to obtain informed consent during this study would be unethical, as it would delay urgent treatment and might lead to additional stress in a patient group for whom this could be harmful. The clinical condition of many patients would also preclude them from absorbing the facts required to make a fully informed decision about study participation. Access to patient data was limited to study researchers and clinicians involved in the care of patients.

Using a nomogram and a standard deviation (SD) of 18 minutes for a previously reported decrease in call to treatment time, it was estimated that nine thrombolysed patients in each group would enable the detection of a 30 minute between groups difference (power of 95\% and $\alpha=0.05) .^{58}$
In the 1 year period before the trial, the SD for call to hospital door times was 27 minutes for patients with chest pain: this was used as a proxy for the SD of call to thrombolysis times, which were unavailable. To provide a baseline to determine if confounding factors had influenced call to thrombolysis times during the trial, we calculated that a sample size of 60 cases was required to yield a pre-trial call to thrombolysis time with a 95\% confidence interval of $+/-7$ minutes.

Analysis was conducted on an intention to treat basis. Point estimates were obtained and $\mathrm{p}$ values calculated by unpaired Student's $t$ test or Mann-Whitney $U$ test using the statistical software package SPSS (version 9; SPSS Inc., Chicago, IL, USA). Statistical significance was set at $\mathrm{p}=0.05$. Point estimates, $\mathrm{p}$ values, and confidence intervals for proportions were calculated using StatsDirect software (version 2.2.1; StatsDirect, UK) as were sensitivity, specificity, and likelihood ratios.

\section{Assignment and masking}

A total of 500 sealed, opaque envelopes were assigned in equal numbers to telemetry or control groups and then randomised by research staff at the beginning of the trial using SPSS. Each was then assigned a unique identifying number and issued in numerical order in blocks of 10 to ambulances participating in the trial. When patients met study inclusion criteria, paramedics selected the lowest numbered envelope remaining and followed the control or telemetry treatment instructions contained within. 


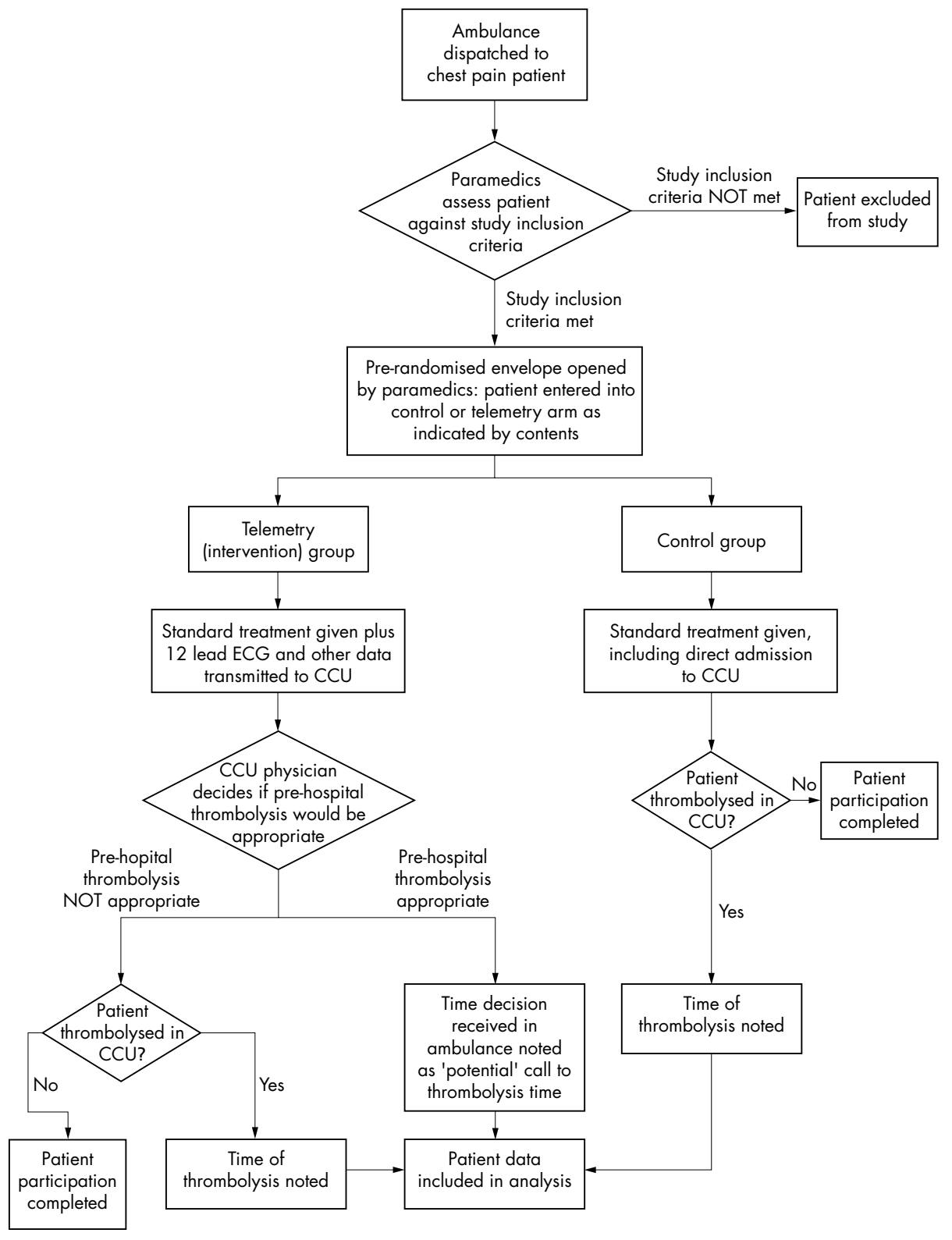

Figure 2 Recruitment process.

Owing to the nature of the study, it was not possible to implement blinding.

\section{RESULTS}

\section{Patient flow and follow up}

Patient recruitment and loss throughout the trial is described in the CONSORT style flow chart (fig 3).

\section{Analysis}

There were no significant demographic differences between the two groups (table 1).

There was no significant difference between mean call to thrombolysis times during the study period compared with the retrospective sample, suggesting that conducting the study had not itself resulted in a change in call to needle times and biased the results (table 2 ).

There were no statistically significant between group differences in call to thrombolysis and on scene times, although the mean journey time to hospital was slightly longer for telemetry patients. The call to door time was significantly longer in the telemetry group as a result of longer response, on scene, and journey times to hospital. The lack of any significant between groups difference in call to needle time is explained by shorter door to needle times in the telemetry group, although this did not reach statistical significance (table 3 ).

There was a potential saving of 55 minutes in call to treatment times for telemetry patients receiving a recommendation for pre-hospital thrombolysis, despite the small numbers of subjects involved and the longer on scene time for telemetry patients (table 4 ).

There was no difference in the call to treatment times between control patients and telemetry subjects thrombolysed in hospital but not receiving a recommendation for pre-hospital intervention. The call to door time was significantly longer in the telemetry group as a result of longer response, on scene, and journey times to hospital. The lack of any significant between groups difference in call to needle time is explained by shorter door to needle times in the telemetry group, although this did not reach statistical significance (table 5). 


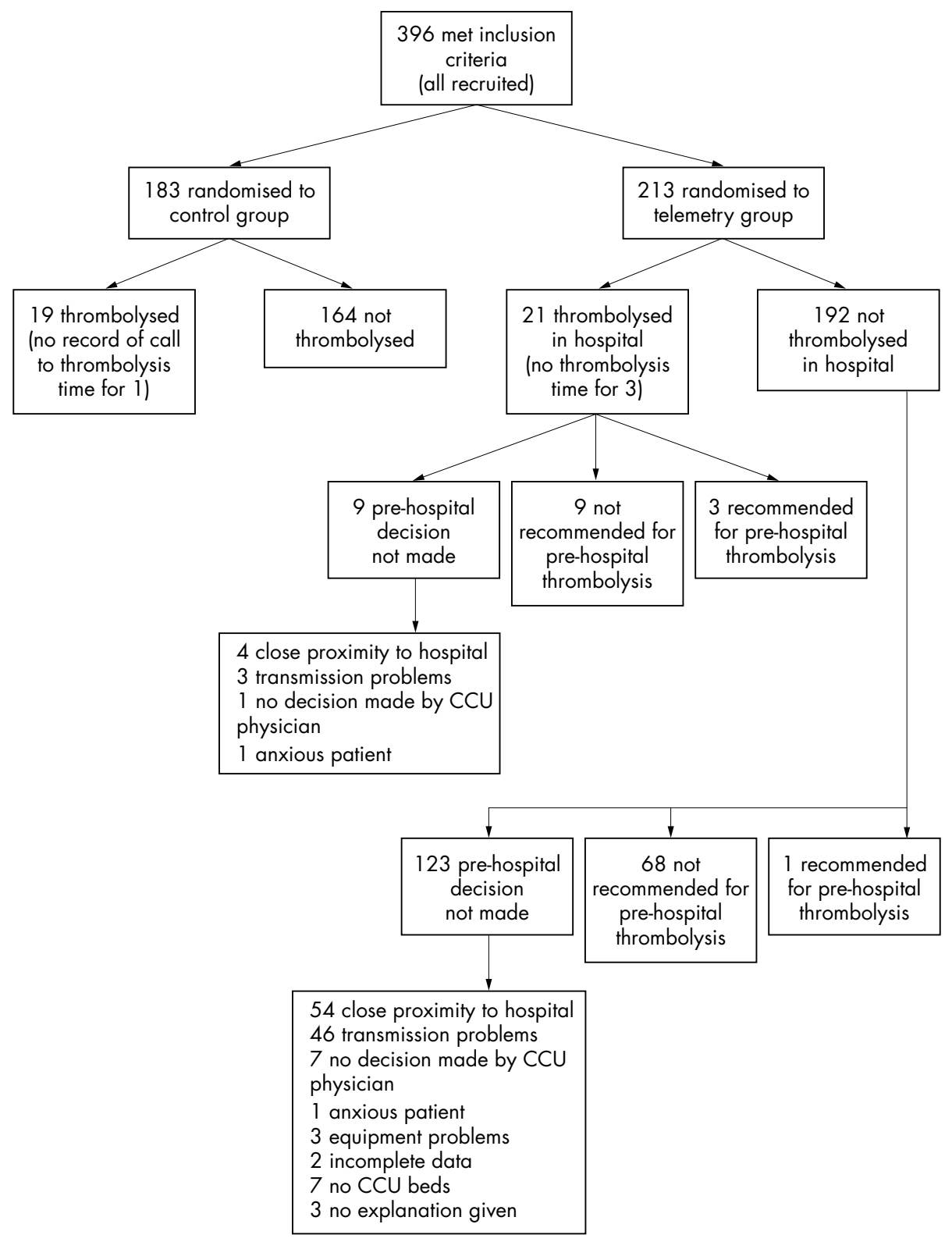

Figure 3 CONSORT style flow chart.

Of the telemetry patients, $21 / 213$ received thrombolytic therapy following hospital admission (10\%; 95\% confidence interval (CI) $6 \%$ to $15 \%$ ). Physicians had recommended pre-hospital thrombolysis for $4 / 213$ telemetry patients (1.9\%; $0.5 \%$ to $4.7 \%$ ), but subsequently, only three were thrombolysed on arrival in the CCU (1.4\%; $0.3 \%$ to $4.1 \%)$. Of the cohort of telemetry patients ultimately receiving thrombolysis, $3 / 21$ received a recommendation for this intervention in the ambulance ( $14 \% ; 3.1 \%$ to $36.3 \%$ ).

One patient recommended for pre-hospital thrombolysis was not administered this treatment following admission to
CCU. The ambulance ECG showed left bundle branch block, but examination of hospital records following admission identified that this condition was not new and that thrombolysis was therefore contraindicated. Had a thrombolytic been available in the ambulance during this trial, inappropriate administration might therefore have occurred.

One telemetry patient was incorrectly identified as not meeting the criteria for pre-hospital thrombolysis. This was due to paramedics incorrectly documenting the blood pressure as being higher than the recommended limit for thrombolysis before transmission to the CCU.

\begin{tabular}{llll}
\hline Table 1 Demographics & & \\
\hline & Control & Telemetry & Difference $(95 \% \mathrm{Cl}, \mathrm{p})$ \\
\hline $\begin{array}{l}\text { Mean age in years (SD) } \\
\text { Proportion of male patients (\%) }\end{array}$ & $113.9(14.0)^{* *}$ & $69.4(14.0) \dagger$ & $1.5(1.3$ to $4.3, \mathrm{p}=0.290)$ \\
\hline Data missing for *one patient, and twwo patients. & $119 / 213(56 \%)$ & $6 \%(-4$ to $16 \%, \mathrm{p}=0.222)$ \\
\hline
\end{tabular}


Table 2 Comparison of mean call to thrombolysis times between retrospective and study control groups

\begin{tabular}{lllll}
\hline & $\begin{array}{l}\text { Retrospective sample }(\mathbf{n}=60) \\
\text { (range, inter-quartile range) }\end{array}$ & $\begin{array}{l}\text { Patients in control arm } \\
\text { receiving thrombolytics } \\
(\mathbf{n}=19+) \text { (range, } \\
\text { inter-quartile range) }\end{array}$ & $\begin{array}{l}\text { Difference } \\
\text { (minutes) }\end{array}$ & p \\
\hline $\begin{array}{l}\text { Median call to needle } \\
\text { time (minutes) }\end{array}$ & 114 (42 to 436,88 to 166) & $108(34$ to 305,63 to 238) & -6 & 0.767 \\
\hline *Data missing for one patient. & & & & \\
\hline
\end{tabular}

Table 3 Between groups comparison of all patients receiving thrombolytic therapy in hospital

\begin{tabular}{|c|c|c|c|c|}
\hline & Control group $(n=19)$ & Telemetry group $(n=21)$ & Difference (minutes) & $\mathbf{p}$ \\
\hline $\begin{array}{l}\text { Median call to thrombolysis time (mins) } \\
\text { (range, inter-quartile range) }\end{array}$ & 108 (34 to 305,63 to 238 ) & 110 (78 to 244,86 to 168$)$ & +2 & 0.715 \\
\hline Mean journey to hospital (mins) $(95 \% \mathrm{Cl})$ & $16(10$ to 21$)$ & $26(20$ to 32$)$ & +10 & $0.013^{*}$ \\
\hline Mean on scene time (mins) $(95 \% \mathrm{Cl})$ & $15(12$ to 19$)$ & $20(15$ to 25$)$ & +5 & 0.106 \\
\hline $\begin{array}{l}\text { Median call to door time (mins) (range, } \\
\text { inter-quartile range) }\end{array}$ & $40(16$ to 80,22 to 56$)$ & $59(22$ to 110,44 to 73$)$ & +19 & $0.018^{*}$ \\
\hline $\begin{array}{l}\text { Median door to thrombolysis time (mins) } \\
\text { (range, inter-quartile range) }\end{array}$ & 77 (14 to 264,19 to 208 ) & $66(19$ to 169,30 to 116$)$ & -11 & 0.730 \\
\hline
\end{tabular}

The sensitivity of the telemetry system in identifying patients suitable for thrombolysis was $13.6 \%$ (95\% CI $2.9 \%$ to $34.9 \%$ ), with a specificity of $99.5 \%$ (97.1\% to $99.9 \%)$. The positive and negative likelihood ratios were 26.1 (3.8\% to $176.0 \%)$ and $0.87(0.67 \%$ to $0.96 \%)$ respectively. This suggests that patients not requiring thrombolysis were unlikely to receive a recommendation for pre-hospital intervention, although a high proportion of eligible patients was missed (table 6).

Of the control group, 19/183 patients were thrombolysed in the CCU $(10.4 \%, 95 \%$ CI $6.4 \%$ to $15.7 \%)$. The between groups difference in the proportion of patients being thrombolysed is not significant $(0.5 \%, 95 \%$ CI -5.5 to $+6.8 \%, p=0.745)$.

\section{DISCUSSION}

Continuous telemetry systems have the potential to reduce call to treatment times for patients with indications for prehospital thrombolysis by 55 minutes. However, the proportion of patients ultimately receiving thrombolytic therapy who receive a recommendation for pre-hospital treatment is small.

The potential saving in call to thrombolysis time is of considerable importance, and compares with a similar UK study." A reduction of 1 hour in call to thrombolysis time results in an additional 21 lives saved per 1000 patients at 35 days. ${ }^{1}$ It has been estimated that every minute of delay to thrombolysis in the first 3 hours of infarction costs, on average, 10 days of life for patients. ${ }^{10}$ If re-perfusion of an occluded coronary artery occurs within 15-30 minutes of symptom onset, it is possible to prevent any permanent damage to the myocardium. ${ }^{11}$ Pre-hospital thrombolysis is likely to be the only means by which treatment can be provided within this challenging time frame.

Telemetry did not increase on scene times to a clinically significant degree, although journey times to hospital were longer in this group. This may have been due to a between groups difference in distance from hospital (we did not record this data) or to the need to stop the ambulance to transmit data when a mobile phone signal was only intermittently available. Despite longer call to door times in the telemetry group, there were no significant delays to treatment for those patients who required thrombolysis but for whom a pre-hospital intervention was not recommended. This appeared to be due to shorter door to needle times in the telemetry group, although this difference was not statistically significant. Previous telemetry research has, however, reported larger reductions in hospital door to thrombolysis times of $20-55$ minutes. ${ }^{6}{ }^{13}$ It is possible that our study's small sample size resulted in a type II error for this variable.

If the increased call to door time noted in the telemetry group is true for all chest pain patients for whom this technology is used (potentially equivalent to $10 \%$ of all 999 calls), the consequent lengthening of the job cycle may result in a requirement to increase the number of ambulances available to maintain response standards. If so, the impact might be particularly significant in urban areas with high call volumes and short response and journey times to hospital, although further research is required to confirm this.

Table 4 Between groups comparison of telemetry patients receiving a recommendation for pre-hospital thrombolysis and control patients thrombolysed in hospital

\begin{tabular}{|c|c|c|c|c|}
\hline & $\begin{array}{l}\text { Control group ( } n=19 \text { ) } \\
\text { (range, inter-quartile range) }\end{array}$ & $\begin{array}{l}\text { Telemetry group patients } \\
\text { receiving a recommendation for } \\
\text { pre-hospital thrombolysis }(n=4) \\
\text { (range, inter-quartile range) }\end{array}$ & $\begin{array}{l}\text { Difference } \\
\text { (minutes) }\end{array}$ & $\mathbf{p}$ \\
\hline Median call to thrombolysis time (mins) & $\begin{array}{l}108 \text { (34 to } 305,63 \text { to } 238 \text { ) } \\
\text { (call to actual thrombolysis time) }\end{array}$ & $\begin{array}{l}53(31 \text { to } 60,35 \text { to } 60) \text { (call to } \\
\text { potential thrombolysis time) }\end{array}$ & -55 & $0.022^{*}$ \\
\hline Median journey to hospital (mins) & 15 (3 to 43,6 to 20$)$ & $29(21$ to 34,21 to 34$)$ & +14 & 0.056 \\
\hline Median on scene time (mins) & $13(6$ to 32,10 to 19$)$ & $21(20$ to 46,20 to 39$)$ & +8 & $0.021^{*}$ \\
\hline
\end{tabular}




\begin{tabular}{|c|c|c|c|c|}
\hline & $\begin{array}{l}\text { Control group }(n=19) \\
(95 \% \mathrm{CI})\end{array}$ & $\begin{array}{l}\text { Telemetry group patients } \\
\text { thrombolysed in hospital butnot } \\
\text { receiving arecommendation for } \\
\text { pre-hospital thrombolysis } \\
(\mathrm{n}=18)(95 \% \mathrm{Cl})\end{array}$ & $\begin{array}{l}\text { Difference } \\
\text { (minutes) }\end{array}$ & $\mathbf{p}$ \\
\hline Mean call to actual thrombolysis time (mins) & 141 (96 to 185$)$ & 141 (109 to 172) & 0 & 0.588 \\
\hline Mean journey to hospital (mins) & $16(10$ to 21$)$ & 25 (19 to 32$)$ & +9 & $0.025^{*}$ \\
\hline Mean on scene time (mins) & $15.3(11.6$ to 18.9$)$ & $18.2(14.0$ to 22.4$)$ & +2.9 & 0.247 \\
\hline $\begin{array}{l}\text { Median call to door time (mins) (range, } \\
\text { inter-quartile range) }\end{array}$ & $40(16$ to 80,22 to 56$)$ & 59 (22 to 110,40 to 74$)$ & +19 & $0.029^{*}$ \\
\hline $\begin{array}{l}\text { Median door to thrombolysis time (mins) } \\
\text { (range, inter-quartile range) }\end{array}$ & 77 (14 to 264,19 to 208 ) & 70 (21 to 169,36 to 130$)$ & -7 & 0.940 \\
\hline
\end{tabular}

Telemetry has other limitations. On rough roads, ambulances had to slow or stop to obtain artefact free ECGs, and it was sometimes necessary to stop in one of a few areas with a good telephone signal to successfully transmit data. Sometimes a treatment decision could not be obtained because of communications problems alone, potentially denying pre-hospital thrombolysis to eligible patients, a finding similar to that of previous studies. ${ }^{42}{ }^{13}$ The cardiology senior house officers (SHOs) were not based in the CCU and consequently there were sometimes delays in obtaining a treatment recommendation. There was an inevitable time lag between the ambulance alerting the CCU about a potential thrombolysis patient and the transmission of eligibility data, frustrating some physicians. Some SHOs used the telemetry link to ask inappropriate questions about the patient's condition, increasing transmission delays by distracting paramedics and blocking communications. Personnel not familiar with computers found it easier to revert to historic treatment regimens, especially when the telemetry connection was not immediate. Rotation of medical staff required new personnel to be trained every 6 months, and there were inevitably periods when no trained physicians were on duty and no treatment decisions were made.

Several physicians expressed concern about prescribing potentially dangerous drug therapy for patients they had not personally examined. These concerns may be amplified in systems that use telemetry to make decisions about actual administration of thrombolytics rather than running in "shadow" form. We found a low likelihood of inappropriate pre-hospital thrombolysis. However, a very low proportion of patients ultimately receiving thrombolytic therapy received a recommendation for pre-hospital treatment (3/21, 14\%). A study in Scotland reported 28/31 patients considered for thrombolysis received pre-hospital treatment using a similar telemetry system $(90 \%, 95 \%$ CI $74-98 \%) .{ }^{9}$ This variability may be due to differences in the experience of the physicians making the treatment recommendation. In our trial, cardiology

\begin{tabular}{|c|c|c|c|}
\hline \multirow{2}{*}{$\begin{array}{l}\text { Recommendation } \\
\text { for pre-hospital } \\
\text { thrombolysis }\end{array}$} & \multicolumn{2}{|c|}{$\begin{array}{l}\text { Thrombolysed } \\
\text { in hospital? }\end{array}$} & \multirow[b]{2}{*}{ Totals } \\
\hline & $\overline{\text { Yes }}$ & No & \\
\hline Yes & 3 & 1 & 4 \\
\hline No & 19 & 190 & 209 \\
\hline Totals & 22 & 191 & 213 \\
\hline
\end{tabular}

SHOs undertook this task, but in Scotland "senior medical staff in the accident and emergency department" made the recommendation. There were also differences in the inclusion criteria for pre-hospital thrombolysis. We limited eligibility to patients <76 years old, used more cautious values for acceptable blood pressure range, included an acceptable range for pulse rate, and excluded patients with a history of a resuscitation attempt or liver or renal failure.

Paramedics acting autonomously have identified patients eligible for thrombolysis with a higher sensitivity than in our trial $(46.7 \%$ versus $13.6 \%)$ but with a similarly safe specificity. ${ }^{14}$ Paramedics in this previous trial attended a 12 hour class; in our study, 18 hours of training were required. Other research has reported that paramedics had a lower sensitivity than "duty emergency physicians" in identifying patients suitable for thrombolysis (71 v 90\%, $\mathrm{p}=0.001)$ but a higher specificity $(97 \vee 94 \%, \mathrm{p}=0.001) .{ }^{15}$

\section{CONCLUSIONS}

Continuous telemetry of 12 lead ECG and clinical data can considerably reduce call to treatment times for patients identified as eligible for pre-hospital thrombolysis in a rural setting. However, its benefits must be balanced against the challenges posed by the training requirements of ambulance and hospital staff, and the small risk of errors in recommending treatment inappropriately. More importantly, continuous telemetry systems may identify only a very small proportion of thrombolysis eligible patients as suitable for pre-hospital treatment. This limitation may be due to communications problems, the criteria used to identify eligible patients, or the seniority of physicians tasked to make treatment decisions.

\section{ACKNOWLEDGEMENTS}

The authors thank the ambulance and CCU staff who participated in this study for their support and expertise; without them, this trial would not have been possible. We are also grateful to Ortivus UK for the provision of telemetry equipment and technical support.

\section{Authors' affiliations}

M Woollard, Faculty of Pre-hospital Care Research Unit, Department of Academic Emergency Medicine, Academic Centre, The James Cook University Hospital, Marton Road, Middlesbrough TS4 3BW, UK K Pitt, A J Hayward, Welsh Ambulance Services NHS Trust, Ty Maes Y Gruffydd, Cefn Coed, Swansea SA2 OGP, UK

N C Taylor, West Wales General Hospital, Glangwili, Carmarthen SA31 2AF, UK

The study was supported by a small project grant from the Wales Office of Research and Development. Ortivus UK supplied, fitted, and maintained the equipment for this study. A satellite phone and the cost of calls in the latter part of the study were funded by ESYS.

Competing interests: there are no competing interests 


\section{REFERENCES}

1 Boersma E, Maas AC, Deckers JW, et al. Early thrombolytic treatment in acute myocardial infarction: reappraisal of the golden hour. Lancet 1996;348:771-5.

2 Department of Health. National service framework for coronary heart disease. London: Department of Health, 2000.

3 National Assembly for Wales. Tackling coronary heart disease in Wales: implementing through evidence. Cardiff: National Assembly for Wales, 2001.

4 Aufderheide TP, Hendley GE, Woo J, et al. A prospective evaluation of prehospital 12-lead application in chest pain patients. J Electrocardiol 1992;24(suppl):8-13.

5 Weaver WD, Eisenburg MS, Hallstrom AP, et al. Myocardial Infarction Triage and Intervention Project - phase 1: Patient characteristics and feasibility of prehospital initiation of thromblytic therapy. JACC 1990;15:925-31.

6 Karagounis L, Ipsen SK, Jessop MR, et al. Impact of field-transmitted electrocardiography on time to in-hospital thrombolytic therapy in acute myocardial infarction. Am J Cardiol 1990;66:786-91.

7 Aufderheide TP, Hendley GE, Keelan MH, et al. Milwaukee Prehospital Chest Pain project - phase 1: Feasibility and accuracy of prehospital thrombolytic candidate selection. Am J Cardiol 1992;69:991-6.
8 Altman DG. Statistics and ethics in medical research. III. How large a sample? BMJ 1980;281:1336-8.

9 Pedley DK, Bisset K, Connolly EM, et al. Prospective observational cohort study of time saved by prehospital thrombolysis for ST elevation myocardial infarction delivered by paramedics. BMJ 2003;327:22-6.

10 Rawles JM. Quantification of the benefit of earlier thrombolytic therapy: fiveyear results of the Grampian Region Early Anistreplase Trial (GREAT). J Am Coll Cardio 1997;30:1181-6.

11 Saltissi S, Mushahwar SS. The management of acute myocardial infarction. Postgrad Med J 1995:71:534-41.

12 Gibler WB, Kereiakes DJ, Dean EN, et al. Prehospital diagnosis and treatment of acute myocardial infarction: a north-south perspective. The Cincinnati Heart Project and the Nashville Prehospital TPA Trial. Am Heart J 1991;121:1-11.

13 Keriakes DJ, Gibler WB, Martin LH, et al. Relative importance of emergency medical system transport and the prehospital electrocardiogram on reducing hospital time delay to therapy for acute myocardial infarction: a preliminary report from the Cincinnati Heart Project. Am Heart J 1992:123:835-40.

14 Pitt K. Pre-hospital selection of patients for thrombolysis by paramedics. Emerg Med J 2002;19:260-3.

15 Keeling P, Hughes D, Price L, et al. Safety and feasibility of prehospital thrombolysis carried out by paramedics. BMJ 2003;327:27-8. 